\title{
An Immune-associated IncRNA Signature Predicts the Survival of Patients With Head and Neck Squamous Cell Carcinoma
}

\section{Jian Wang}

Xinjiang Medical University

Qinjiang Bian

: Gansu Provincial Hospital

Jialin Liu

Xinjiang Medical University

Lijuan Du

Urumqi Vocational University

Adili Moming ( $\sim 2408831296 @ q q . c o m$ )

Xinjiang Medical University

\section{Research Article}

Keywords: head and neck squamous cell carcinoma, Immune, Long non-coding RNA, Prognosis,

Signature

Posted Date: April 27th, 2021

DOl: https://doi.org/10.21203/rs.3.rs-446589/v1

License: (c) (i) This work is licensed under a Creative Commons Attribution 4.0 International License. Read Full License 


\section{Abstract}

Recent researches have established that IncRNAs (long non-coding RNAs) could be exploited as new signatures for head and neck squamous cell carcinoma (HNSCC) diagnosis, prognosis, and treatment. Herein, HNSCC transcriptome data was abstracted from the Cancer Genome Atlas (TCGA) data resource, and uncovered immune linked IncRNAs through co-expression analysis. Besides, univariate along with Lasso penalty regression were employed to determine immune-linked IncRNA pairs with different expressions. We then compared area under the curve, calculated the Akaike information criterion (AIC) value of the receiver operating characteristic curve for 5 years, determined cutoff points, and established an optimal predictive model for identifying high- and low-risk HNSCC patients. Overall, we identified 40 differentially expressed immune-linked IncRNA pairs, 17 of which were incorporated in the Cox regression model. Using this model, we can more effectively stratify patients based on poor survival results, positive clinicopathological features, specific tumor immune invasion status, low chemotherapy responsivity, and high expression of immunosuppressive biomarkers. Our data illustrated that the immune-linked IncRNA pairs signature have clinical prediction value for HNSCC.

\section{Full-text}

Due to technical limitations, full-text HTML conversion of this manuscript could not be completed. However, the manuscript can be downloaded and accessed as a PDF.

\section{Figures}



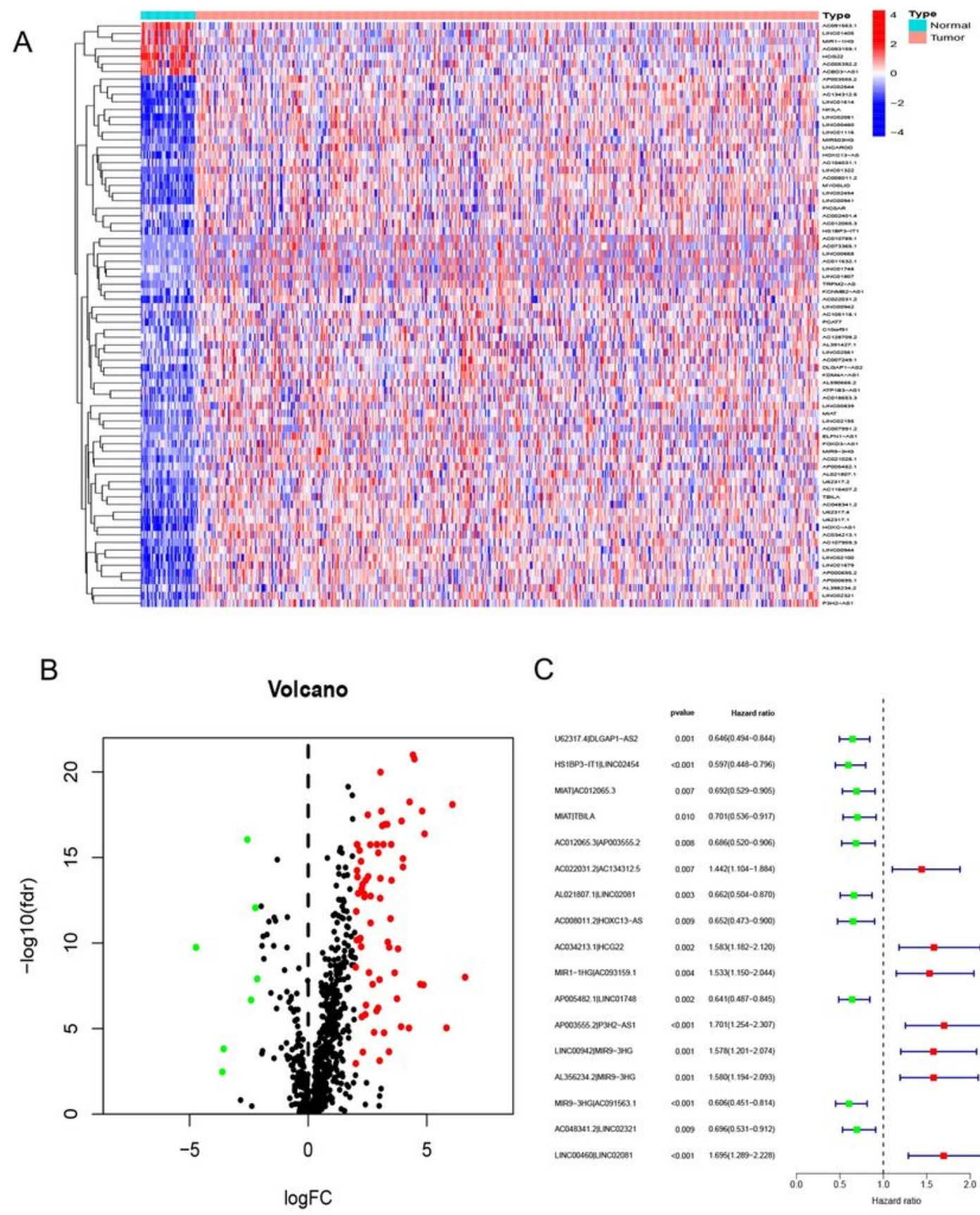

C

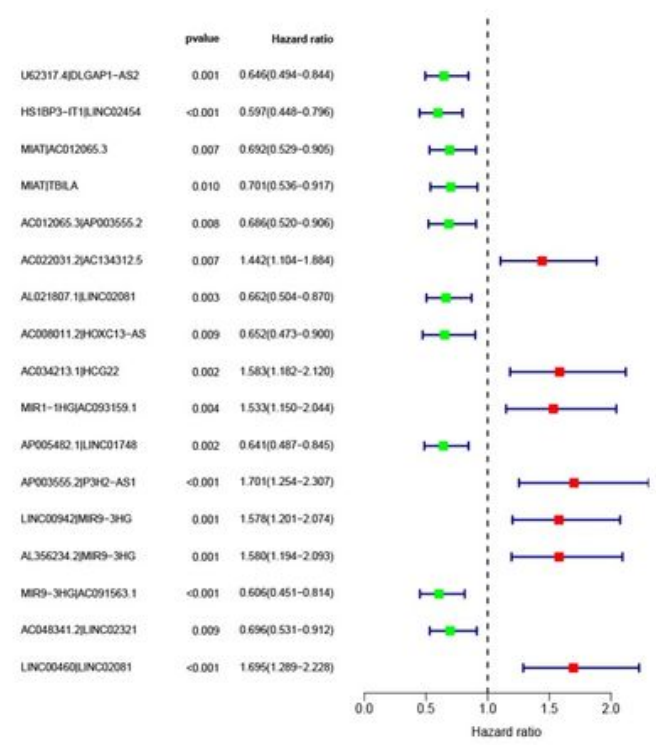

\section{Figure 1}

DeirlncRNA Pairs were used to establish a risk assessment model Using TCGA data set and Ensembl annotation to identify differentially expressed immune-related IncRNAs (DEIRLNcRNAs) (A, B). (A) Heatmaps of DeirlncRNAS for HNSCC and non-tumor (control) tissues. (B) Volcano plot shows the DeirlncRNAS. (C) The forest map showed 17 pairs of deirlncRNAs, identified by Cox proportional hazard regression in the stepwise method. 
A

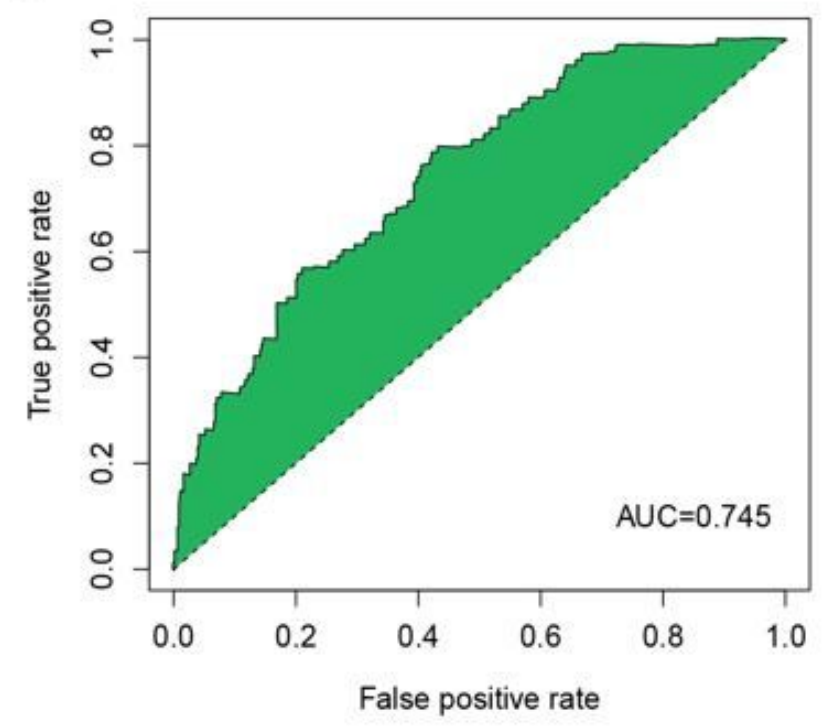

C

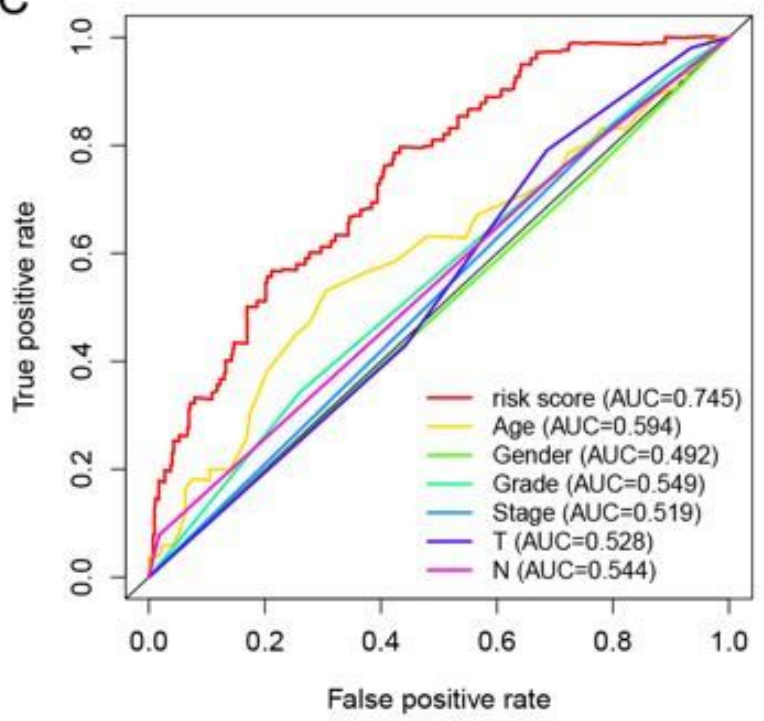

B

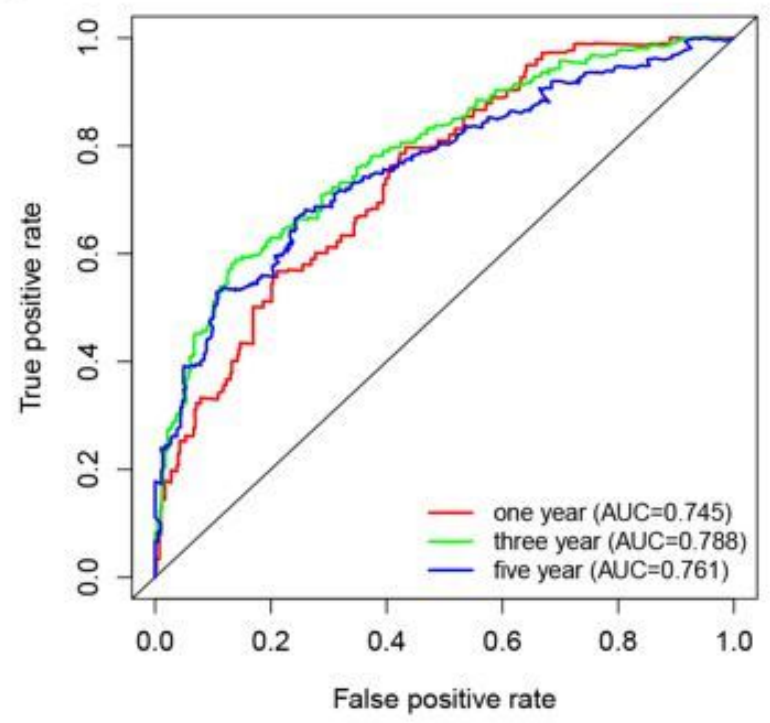

D

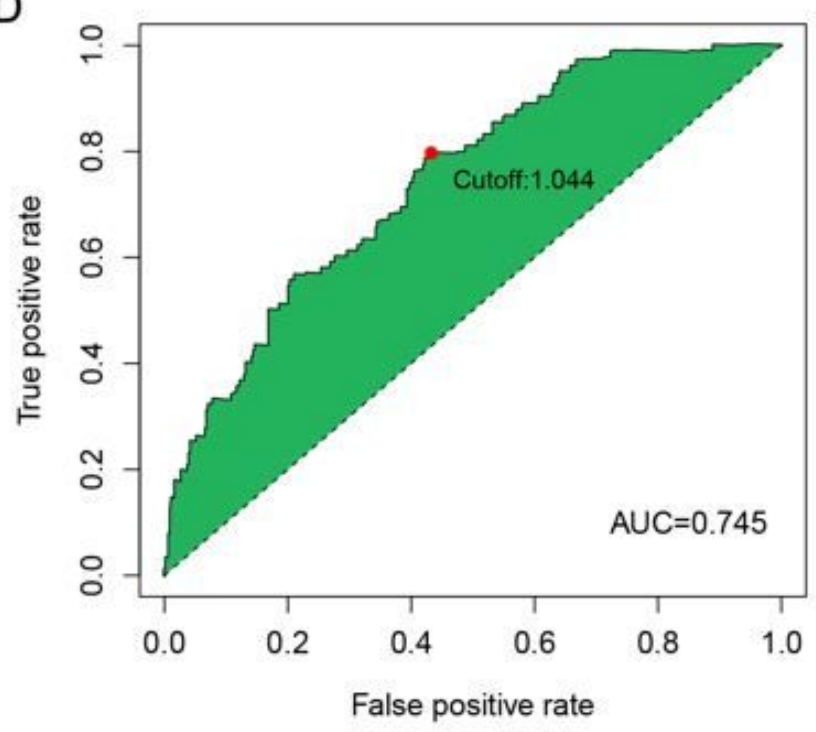

Figure 2

DeirlncRNA Pair were used to establish a risk assessment model (A) The maximum AUC value of the optimal deirlncRNA pairing model. (B) The ROC curves of the optimal model for 1-, 3- and 5-years showed that all AUC values were over 0.74. (C) The 5-year ROC curve was compared with other common clinical features, showed the superiority of Riskscore. (D) Risk score of 545 patients with HNSCC; the cutoff point obtained by AIC is the maximum inflection point. 
A

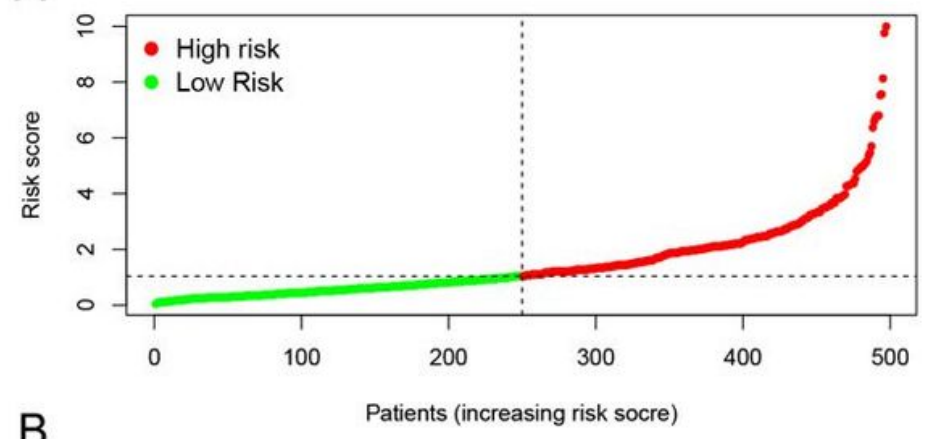

B

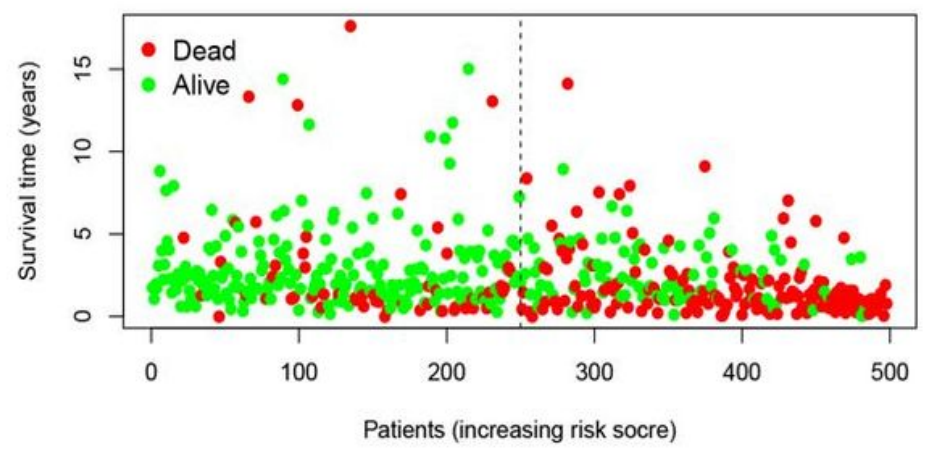

C Risk \pm High risk $\mp$ Low risk

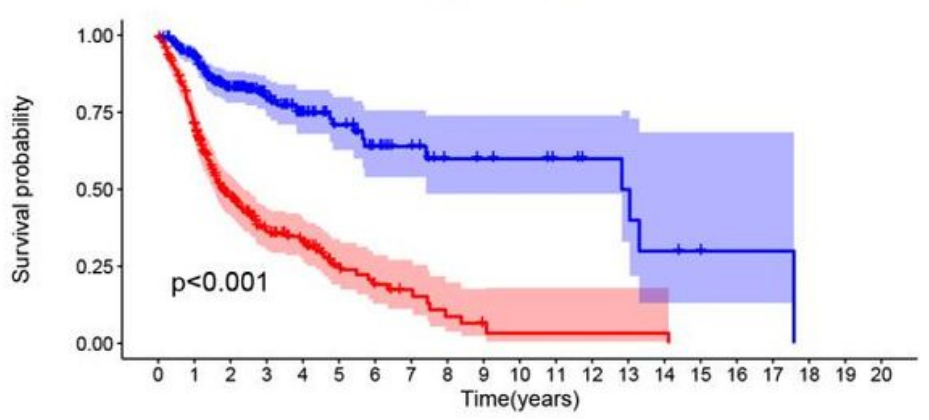

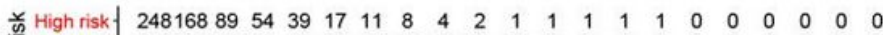
帝 Low risk \begin{tabular}{|cccccccccccccccccccccc}
250209126 & 80 & 54 & 35 & 23 & 18 & 12 & 11 & 10 & 8 & 6 & 5 & 3 & 2 & 1 & 1 & 0 & 0 & 0 \\
\hline 0 & 1 & $\dot{2}$ & $\dot{3}$ & 4 & 5 & $\dot{6}$ & $\mathbf{7}$ & $\dot{8}$ & $\dot{9}$ & 10 & 11 & 12 & 13 & 14 & 15 & 16 & 17 & 18 & 19 & 0
\end{tabular} Time(years)

Figure 3

Risk assessment model for predicting the prognosis of HNSCC patients (A) The number of patients in high- and low- risk groups. (B) Survival status distribution of high- and low-risk patients. (C) Kaplan-Meier OS curves were constructed for low- and high-risk HNSCC patients. 


\section{A}

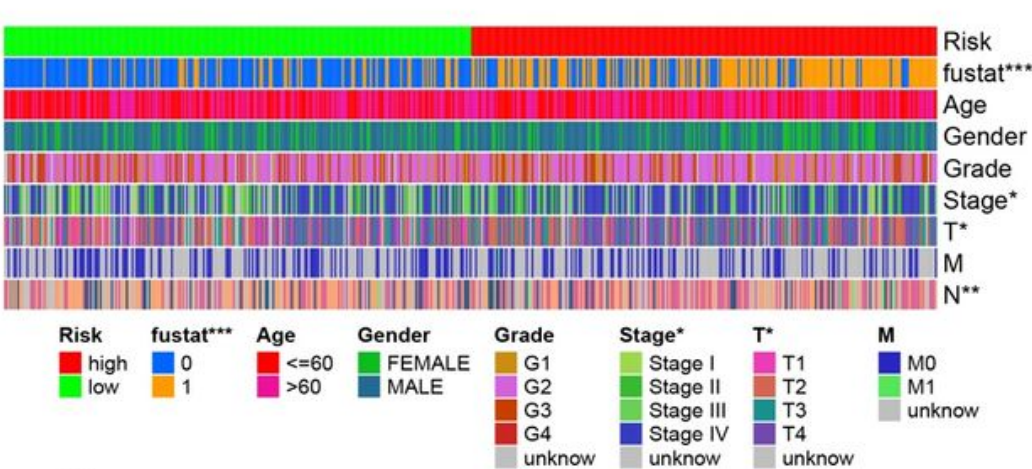

$N^{* *}$

unknow unknow

No

$\mathrm{N} 1$
$\mathrm{~N} 2$
$\mathrm{~N} 3$

unknow

B

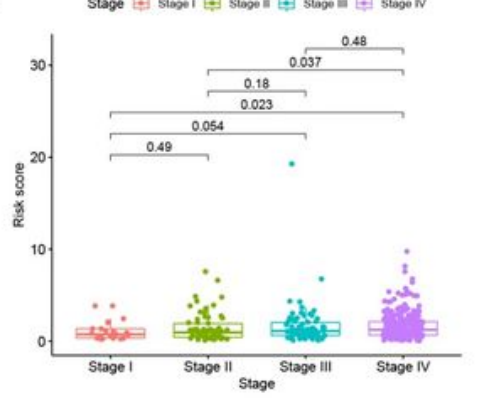

D

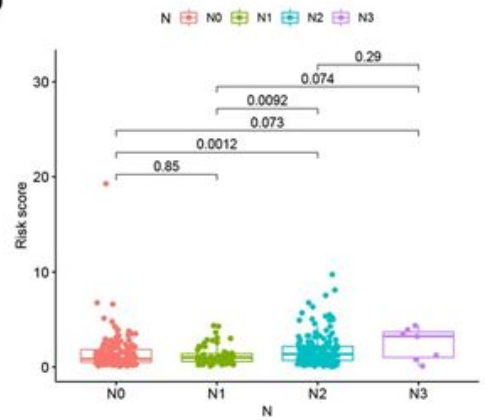

C

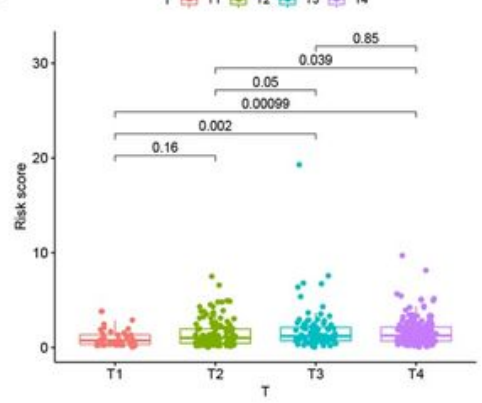

E

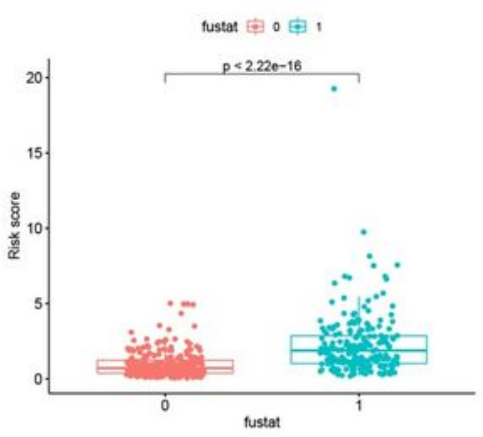

\section{Figure 4}

The clinical features were evaluated by a risk assessment model $A$ strip chart $(A)$ and the scatter diagram showed that (B) clinical stage, (C) T stage, (D) N stage, and (E) survival status were signifificantly associated with the riskscore. 

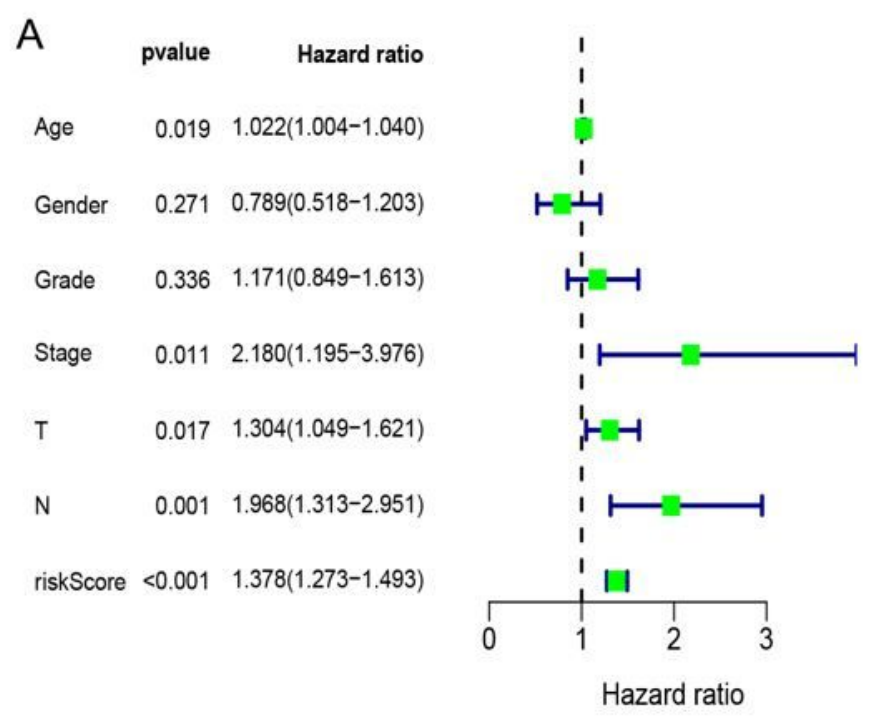
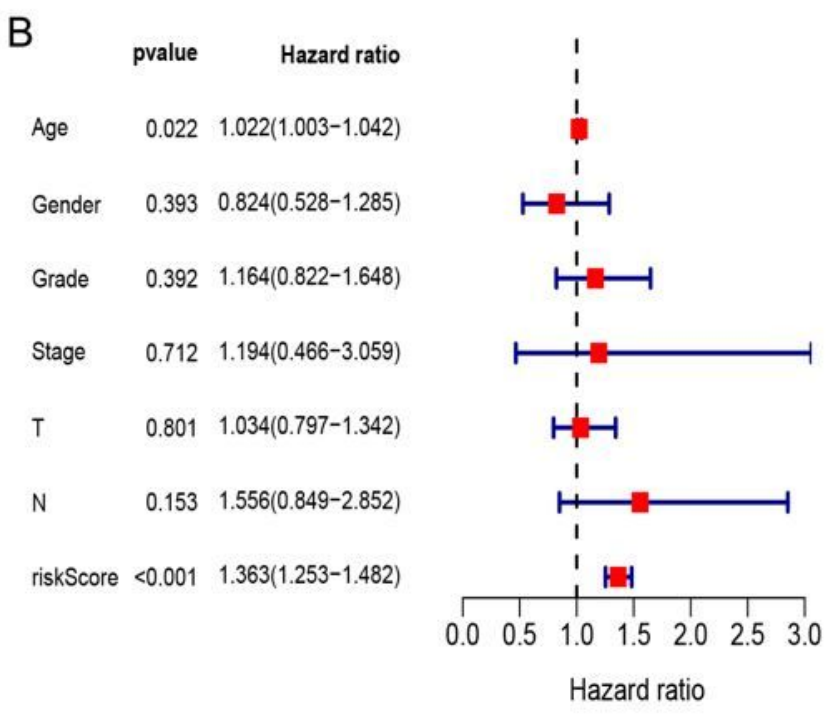

\section{Figure 5}

Univariate and multivariate Cox regression analysis (A) Univariate Cox regression data demonstrated that age $(p=.05, H R=1.022,95 \% \mathrm{Cl}$ [1.004-1.040]), clinical stage ( $p<.05, \mathrm{HR}=2.180,95 \% \mathrm{Cl}$ [1.195-3.976]), T stage $(p<.05, \mathrm{HR}=1.304,95 \% \mathrm{Cl}[1.049-1.621]), \mathrm{N}$ stage $(\mathrm{p}<.01, \mathrm{HR}=1.968,95 \% \mathrm{Cl}$ [1.313-2.951]), Riskscore (HR=1.378, $p<.001,95 \% \mathrm{Cl}[1.273-1.493])$ had a significant difference. (B) Riskscore $(\mathrm{p}<.001$, $\mathrm{HR}=1.363,95 \% \mathrm{Cl}[1.253-1.482])$ and age $(\mathrm{p}<.05, \mathrm{HR}=1.02295 \% \mathrm{Cl}[1.003-1.042])$ were predictors in multivariate Cox regression analysis. 
A

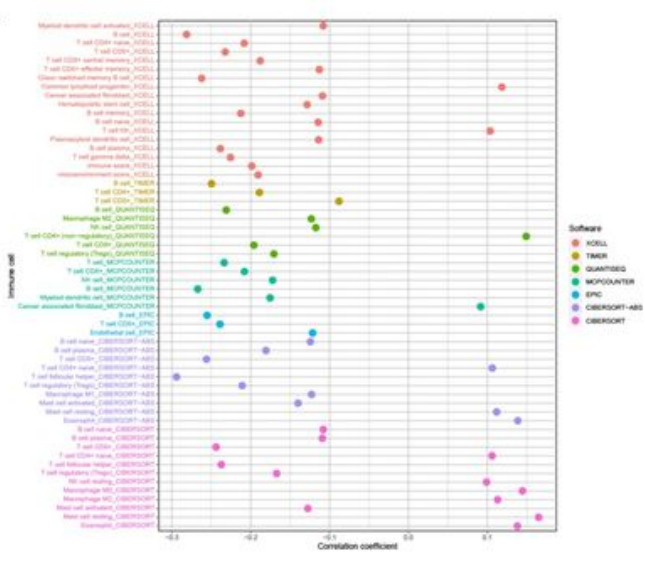

C

Risk $\square$ low $\square$ igh

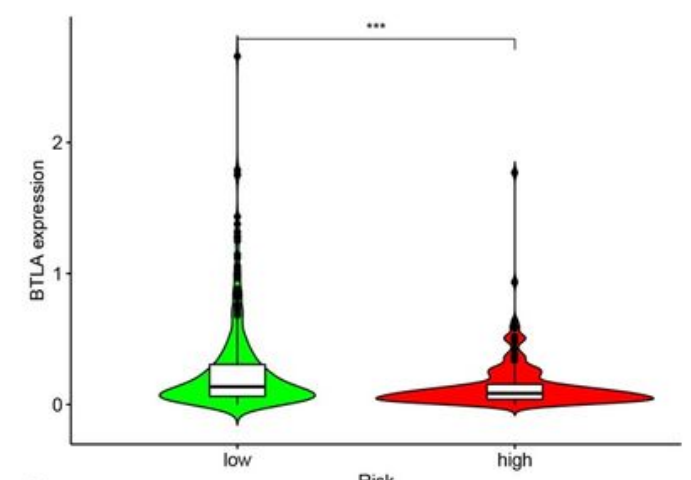

E
B $\quad$ Risk $^{\text {low }} \square_{\text {nigh }}$

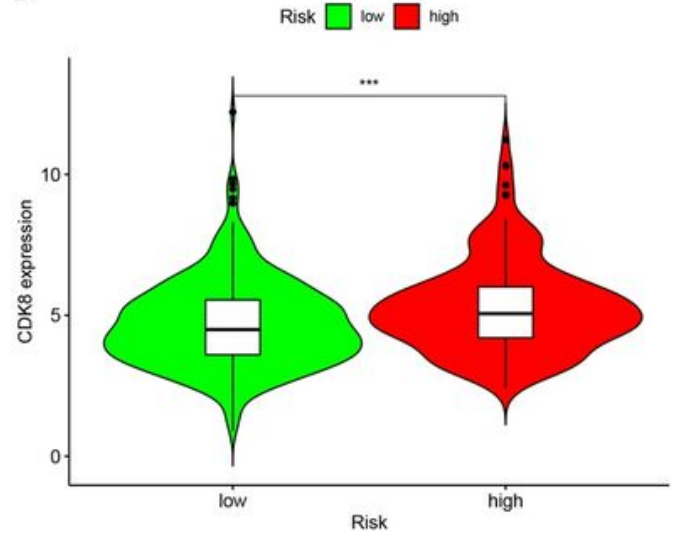

D

Risk $\square$ low $\square$ high

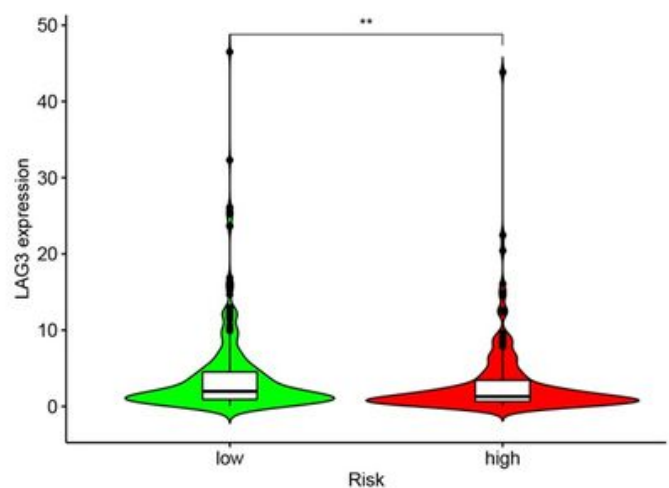

Risk $^{\text {low }} \square_{\text {high }}^{\text {ign }}$

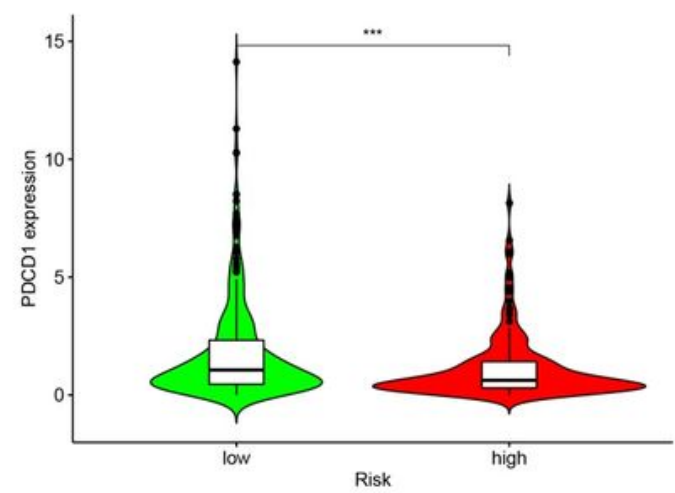

\section{Figure 6}

Evaluation of tumor-invading immune cells and immunosuppressive molecules with risk evaluation model (A) The high- and low risk groups were correlated with tumor invading immune cells like macrophages, CD8+ T-cells, monocytes, along with CD4+ T-cells. High risk scores were positively correlated with high CDK8 expression $(\mathrm{p}<.001, \mathrm{~B})$ and negatively correlated with BTLA , LAG3 and PDCD1 $(p<.001, C-E)$. 
A

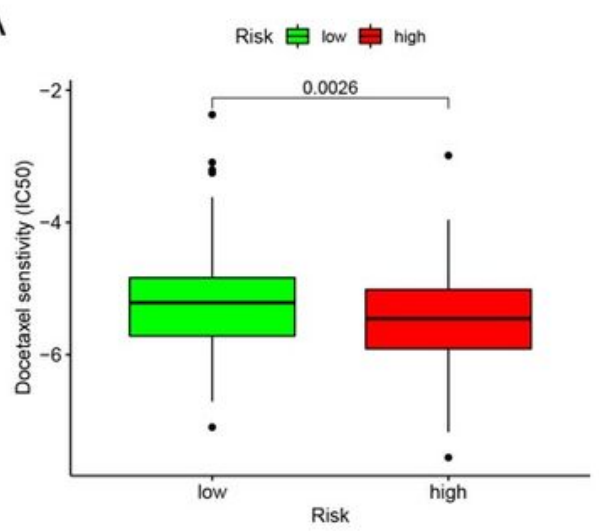

C

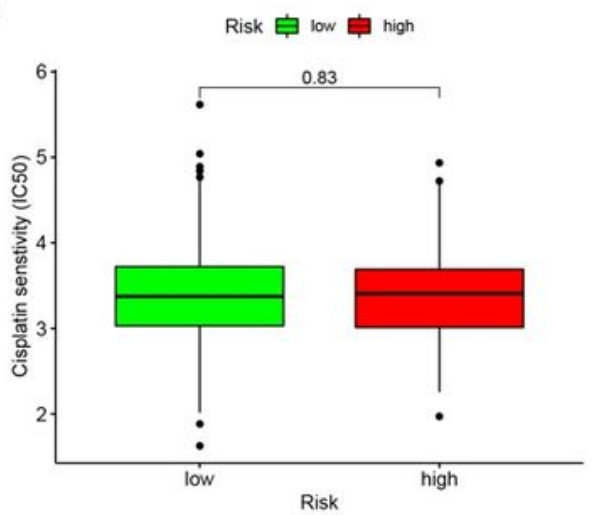

$\mathrm{E}$

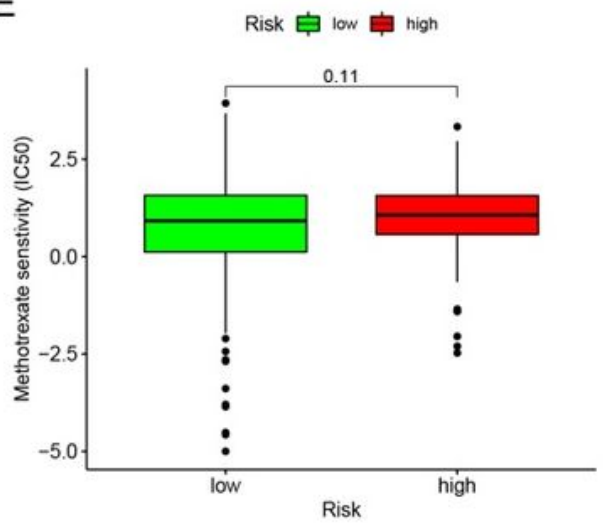

B

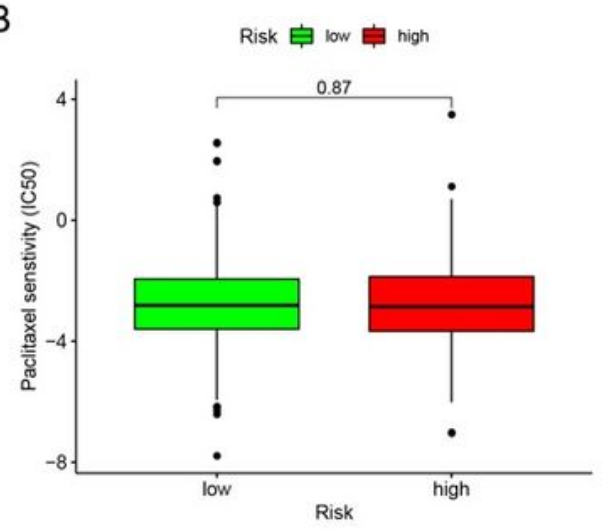

D

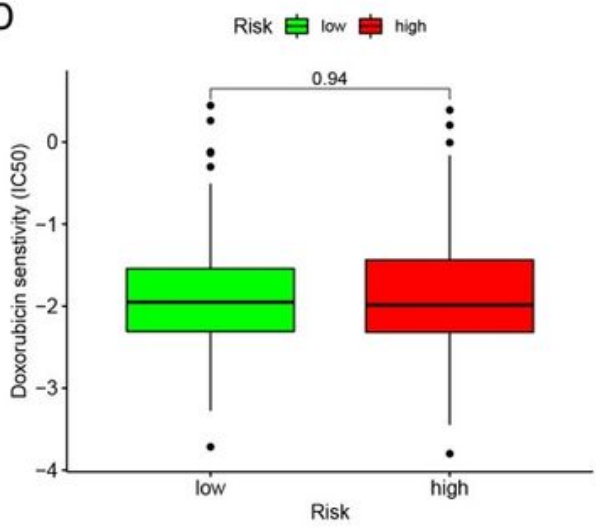

\section{Figure 7}

Correlation analysis of risk model and chemotherapeutics High-risk scores correlated with lower IC50 for Docetaxel $(p<.01, A)$, Risk score was not significantly associated with IC50 for chemotherapeutics such as paclitaxel, cisplatin, doxorubicin, and methotrexate (B-E). 